\title{
Editorial
}

\section{The routes of emergence of life from LUCA during the RNA and viral world}

\author{
Sohan Jheeta \\ NoR HGT \& LUCA, 1 Scott Hall Crescent,Leeds, LSRB7 3,UKe-mail: sohan7@ntlworld.com
}

The aim of Network of Researchers on Horizontal Gene Transfer and the Last Universal Cellular Ancestor (NoR HGT \& LUCA's) is to study the origin of life on Earth by reviewing the role played by HGT during the tweaking of the LUCA up to the point of emergence of the three domains of life.

Why is HGT important? It allows us to work out the genotypes of highly conserved genes (e.g. RNase P) which were present when life 'burst' into Archaea and Bacteria as well as Eukarya (this being the chimera of the first two organisms). Using HGT, it is possible to construct a tree (or 'bush') of life, showing the interconnection of genes between the three domains especially in relation to the commonly shared and conserved ones. Taken together with other studies (e.g. the chemistry of RNA which culminated into a RNA world hypothesis) and our knowledge about viruses, it has been possible to predict the presence of both the LUCA and pre-LUCA. It is thought that the former organism was an almost fully formed cellular entity; the latter's full chemical nature has not yet fully been established, but what has been posited is that there must have been a community made up of many pre-LUCAs which were composed of varying degrees of genetic code, including RNA. Both LUCAs and pre-LUCAs are being surrounded by a membrane vesicle. Additionally, some scientists posit that there may have been three types of pre-LUCA which led to the formation of the eventual three domains of life.

The motive behind forming the network, NoR HGT \& LUCA was to delve further into these hypotheses and elucidate the possible genome of LUCA(s).

This special issue of the IJA is the synopsis of the NoR HGT \& LUCA meeting held at the University of Leeds, UK on the 28-29th October 2014. Some of the papers for this special issue are drawn from that meeting. In this endeavour, I am grateful to Professor Rocco Mancinelli, Editor-in-Chief, of the International Journal of Astrobiology for accommodating the idea. 